\title{
Universidad de Aysén: Nuevos desafíos y oportunidades regionales para la educación chilena
}

\author{
Felipe Rivera Polo ${ }^{1}$, Pablo Rivera-Vargas ${ }^{2}$, Cristina Alonso-Cano ${ }^{3}$
}

\section{Resumen}

La Universidad de Aysén es la primera institución universitaria pública creada en Chile en los últimos 68 años, asumiendo desde su comienzo, una nueva lógica organizativa que se aparta del paradigma convencional con el que se ha configurado el sistema universitario chileno. Su apuesta y compromiso por el desarrollo local y regional, además de la implicación activa de la comunidad, nos hacen prever que su emergencia podría acarrear múltiples transformaciones sociales, tanto en la arquitectura del sistema educativo chileno, como en el centralismo y su insensibilidad por los estímulos territoriales autónomos.

El presente artículo es una reflexión en base a los antecedentes empíricos y bibliográficos disponibles, que más que cerrar el foco analítico del problema, busca promover su análisis y reflexión en la comunidad académica.

Palabras clave: Cambio Educativo, Universidad, Desarrollo Regional, Reforma Educativa, Inclusión Educativa.

\section{I.- Una nueva universidad en Chile: Antecedentes generales}

El 3 de agosto del año 2015 fue promulgada la ley 20.842 que crea las Universidades Públicas de las Regiones ${ }^{4}$ de $\mathrm{O}^{\prime}$ Higgins y de Aysén, en dos

${ }^{1}$ Chileno, Antropólogo. Doctorando en Educación y Sociedad por la Universidad de Barcelona. Investigador de la Biblioteca del Congreso Nacional (Chile). frivera@bcn.cl

${ }^{2}$ Chileno, Sociólogo. Doctor en Educación y Sociedad por la Universidad de Barcelona. Profesor Departamento de Didáctica y Organización Educativa, Universidad de Barcelona. pablorivera@ub.edu

3 Española, Pedagoga. Doctor en Educación por la Universidad de Barcelona. Profesor Departamento de Didáctica y Organización Educativa, Universidad de Barcelona. cristina.alonso@ub.edu

${ }^{4}$ Dice relación con las Divisiones Administrativas, que designan entidades políticas que funcionan como divisiones de un territorio estatal, que tienen carácter formal que se expresa en instituciones y una organización administrativa y política. En el caso de la Unión Europea, siguiendo la Nomenclatura de Unidades Territoriales Estadísticas (NUTS), las Divisiones Administrativas Mayores (DAM) que corresponderían a la escala 
regiones donde no existía una. Este hecho reviste una alta significación, pues hace 68 años que el Estado no creaba una universidad pública $y$, con anterioridad, sólo había creado dos: la Universidad de Chile (1842) y la Universidad Técnica del Estado (1947). Las otras universidades públicas actualmente en existencia (14 instituciones) no surgen como proyectos específicos, sino que en 1981 el gobierno militar de Augusto Pinochet, separó las sedes regionales de estas dos universidades nacionales, adquiriendo autonomía de facto, pero sin una identidad regional en su origen.

El hecho de que estas dos universidades públicas surjan como proyectos regionales desde su génesis, las transforma en un hecho único en la historia universitaria de Chile, pues es primera vez que el Estado es receptivo a la demanda de las regiones por universidades comprometidas con su territorio y su desarrollo, reactividad que ha sido expresión histórica del acerado carácter centralista del Estado chileno (Boisier, 2000). Al mismo tiempo, dicho proceso de creación y puesta en marcha de estas universidades no tiene antecedentes que las precedan, por lo que deben aprender en el hacer y tratar de resistir las enormes fuerzas centralistas del sistema educacional chileno.

Estos antecedentes transforman a este proceso en un fenómeno emergente de gran significatividad, pues existe una creciente demanda de los territorios por una mayor descentralización, y por lo tanto, por una mayor vinculación con sus universidades regionales (Valenzuela, 2015), lo que debería tener prontamente impactos en la arquitectura del sistema de educación superior chileno.

Este proceso de demanda por una mayor vinculación entre universidad y territorio, se da en un contexto de alta movilización social y política, que se expresa en una serie de hechos que enmarcan la creación de estas dos instituciones académicas:

a) Un fuerte cuestionamiento al sistema de educación superior universitario por su carácter de mercado y centralista, que ha generado fuertes presiones para que la educación superior constituya un derecho social garantizado (Atria, 2014).

b) Creciente presión de las regiones por mayor vinculación y pertinencia de las universidades de regiones con su territorio, la que se ha consolidado como una demanda transversal en las regiones del país

de Regiones en Chile sería la NUTS1, que en el caso de España son el Grupo de Comunidades Autónomas. 
(Valenzuela, 2015).

c) La progresiva toma de conciencia de los habitantes en regiones de que la educación representa uno de los elementos claves para que los territorios regionales puedan encarar con éxito el desafío de la sociedad del conocimiento (OCDE, 2009a), potenciando sus ventajas comparativas a nivel regional, nacional y global (Von Baer,2006 y 2007).

\section{El caso de la nueva Universidad de Aysén.}

La Universidad de Aysén responde a que ésta presenta una serie de particularidades que parecieran señalar un cambio de paradigma respecto del resto de las instituciones académicas que conforman el sistema de educación universitaria de Chile, lo que justificaría un estudio en profundidad ${ }^{5}$.

Dentro de estas particularidades cabe mencionar que la Universidad de Aysén surge de una demanda social regional en el año 2012, cuando la población aysenina estableció una serie de exigencias al Estado chileno para deponer su movilización social autodenominada "Aysén, tu problema es mi problema". Una de dichas exigencias era la creación de una "Universidad pública regional de alta calidad y formación académica nacida de los intereses y necesidades de los ayseninos"6, que se entronca con la reivindicación histórica de la Patagonia por "recursos por excepcionalidad y un modelo de desarrollo propio" (Valenzuela 2015: 17).

Esta demanda fue recogida por la Presidenta Michelle Bachelet en su programa de gobierno (Bachelet, 2013: 21), quien al ganar las elecciones en 2013 materializó dicha promesa de campaña en una política pública al promulgar el 2015 la ley no 20.842 que creó la Universidad de Aysén?. Ello implicó que esta universidad tuviera desde

5 La Universidad de la Región de O'Higgins no ha sido seleccionada para esta investigación, por el hecho de encontrarse a mucha cercanía de Santiago, la Capital de Chile (99 kilómetros en carretera), por lo que Rancagua incluso es lugar de residencia de muchos trabajadores de Santiago, por lo que existen posibilidades que termine siendo parte del sistema de universidades de la metrópolis, a diferencia de Aysén, que por su lejanía, emerge la necesidad de autonomía.

6 Pliego de demandas del movimiento social "Aysen, mi problema es tú problema", 15 de febrero de 2012, en Archivo Chile. Disponible en línea en fecha de consulta 20/05/2016:

http://www.archivochile.com/carril_c/cc2012/cc2012-027.pdf

${ }^{7}$ Como también creó la Universidad regional de O’Higgins. 
su génesis la implicación de la comunidad en su génesis, por lo que las aspiraciones regionales deben ser parte fundamental en su puesta en marcha y funcionamiento.

La creación de estas dos nuevas universidades regionales no fue el único compromiso en materia de educación superior de Bachelet, quien asumió la responsabilidad de llevar a cabo una profunda reforma al sistema de educación superior, que debiera consagrar el derecho social a la educación mediante su gratuidad (Atria, 2014). En este escenario de transformación, la Universidad de Aysén, a través de su Rectora Roxana Pey, plantea ser la primera universidad pública $100 \%$ gratuita, lo que viene a reafirmar el carácter singular y de quiebre con el paradigma del sistema de educación superior universitario chileno de preeminencia del mercado. En palabras de la Rectora Pey: "esta es una universidad que nace no en la lógica de mercado, sino en la lógica de la educación superior como un derecho, por lo tanto, es una universidad que requiere gratuidad, nacer con gratuidad para todos sus estudiantes, y vamos a trabajar durante el 2016 para que eso se logre" ${ }^{8}$.

La relación permanente que ha desarrollado la Universidad de Aysén con la región, va más allá de su génesis, ya que la comunidad ha participado activamente en el diseño y su puesta en funcionamiento. De esta forma, esta es la primera institución pública del sistema universitario de Chile que establece un dialogo permanente con su comunidad y territorio.

Es conveniente remarcar esta última idea de territorio, pues la Universidad de Aysén se encuentra en una de las regiones más aisladas y extremas del país, en plena Patagonia, una zona de fiordos que no tiene conexión terrestre con el resto del país. Es uno de los medioambientes más frágiles del mundo con una serie de especies endémicas, con escasa densidad poblacional -sólo 98.413 habitantes para una superficie de $108494 \mathrm{~km} 2$ (INE, 2012)- y con una de las reservas de agua potable más grandes del planeta después de la antártica, que actualmente se encuentra tensionado por el impacto de inversiones y en materia de energética, silvoagropecuarias, acuícolas y de crecimiento urbano.

\footnotetext{
8 Pey, Roxana (2016), "Rectora de la Universidad de Aysén y sistema educacional: "El Estado es simplemente una caja pagadora a los privados". En El Mostrador, 10 de marzo de 2016. Disponible en línea en fecha de consulta 15/06/2016:

http://www.elmostrador.cl/noticias/pais/2016/03/10/rectora-de-la-universidad-deaysen-y-sistema-educacional-el-estado-es-simplemente-una-caja-pagadora-a-losprivados/
} 
En el proceso de puesta en marcha de la Universidad de Aysén, que recibirá a sus alumnos a partir 2017, la vocación regional se ha materializado en diversos aspectos:

a) Gobierno Universitario:

- Primera universidad chilena que integra permanentemente a la comunidad en el gobierno universitario, a través de un órgano denominado Consejo Social, el cual "...resguarda la pertinencia y compromiso de la institución con su contexto histórico y social, como elemento consustancial a la búsqueda de excelencia y de calidad" (Universidad de Aysén, 2016: 2).

- Además, el Consejo Social tiene un representante en el Consejo Superior de la universidad, que consagra la participación vinculante de la comunidad en el órgano de conducción institucional.

b) Gestión Universitaria:

- El diseño y puesta en marcha de la universidad se ha llevado a cabo en un diálogo directo y permanente con diferentes actores regionales, el cual ha venido desarrollándose de manera sistemática desde el año 2014 (MINEDUC 2015a y 2015b) y continúa realizándose.

- Ha diseñado un mecanismo de ingreso a la universidad que bonifica a los estudiantes residentes de la región: "Consideraremos además, incentivos para que los estudiantes de la región puedan acceder a su propia Universidad estatal y permanezcan en la región" ${ }^{\prime 9}$.

c) Proyecto Educativo:

- Reconoce un "sello regional" en su programa académico que dice relación con una mirada basada en "la conservación del ecosistema, la preocupación por sus habitantes, el desarrollo del turismo y el fortalecimiento de valores éticos y sociales ${ }^{10}$, lo que hace eco de las demandas de su comunidad al respecto.

\footnotetext{
9 Pey, Roxana (2016). "Región y reforma de educación superior". En Universidad de Aysén, 01 de junio de 2016. Disponible en línea en fecha de consulta 15/06/2016: http://www.uaysen.cl/2016/06/01/region-reforma-educacion-superior/

10 Pey, Roxana (2016). "iPartimos con 10 carreras!". En Universidad de Aysén, 18 de mayo de 2016. Disponible en línea en fecha de consulta 20/05/2016:

http://www.uaysen.cl/2016/05/18/partimos-10-carreras/
} 
- Sus 10 carreras proyectadas ${ }^{11}$, responden a las necesidades de profesionales que presenta a día de hoy la región; en palabras de la rectora Pey : "No elegiré las carreras a dedo. Miraremos las preferencias de los jóvenes, pero también en las necesidades de la región y las oportunidades laborales que se ofrecen"12.

- Impartirá una carrera de marcado sello innovador para la oferta chilena, como es el caso de Ingeniería en Energía, que responde a una área de desarrollo estratégico regional, como es potencial de generación eléctrica.

d) Programa de Investigación Científica y Tecnológica:

- Declara que los programas de investigación científica y tecnológica buscarán impacto regional, pero sin dejar de observar las proyecciones nacionales e internacionales de ésta: "La lógica de pertinencia regional nos obliga a definir nuestras carreras de esta forma, relacionadas a la investigación básica y a la investigación aplicada, con proyección internacional pero también nacional y, más importante aún, con impacto local inmediato"13.

Todo ello justificaría el carácter emergente, novedoso y, por lo mismo, no estudiado en Chile. Sus efectos en el sistema universitario chileno, por lo tanto, podría impactar a otras universidades públicas de regiones y al sistema de educación superior chileno en su conjunto. Todo ello justificaría su selección como caso de estudio. De no ser exitoso, igual resulta relevante, ya que entregaría una aproximación a los riesgos que habría que tener presente para vincularse regional y territorialmente en un sistema sometido a fuerzas de centralizadoras.

\section{II.-EI Sistema Universitario Chileno: Desarrollo conceptual y empírico}

11 En 5 áreas como son: Ingeniería (en Energía y Industrial), Ciencias Naturales (Forestal y Agronomía), Salud (Obstetricia y Enfermería), Educación (Primaria y Especial), Ciencias Sociales (Trabajo Social y Administración Pública).

12 Pey, Roxana /2015). "Rectora Roxana Pey: La Universidad de Aysén abrirá sus puertas el 2017". En Radio Universidad de Chile, 21 de septiembre de 2015. Disponible en línea en fecha de consulta 12/03/2016:

http://radio.uchile.cl/2015/09/21/rectora-roxana-pey-la-u-de-aysen-abrira-en-2017

13 Pey, Roxana (2016). "Universidad de Aysén desarrollará seminario académico para definir carreras y planes de estudio". En Universidad de Aysén, 11 de abril de 2016. Disponible en línea en fecha de consulta 09/05/2016:

http://www.uaysen.cl/2016/04/11/universidad-aysen-desarrollara-seminario-

academico-definir-carreras-planes-estudio/ 


\section{EI Sistema de Educación Superior Universitario chileno.}

El esquema 1 evidencia los tipos de universidades existentes en Chile, y que conforman la arquitectura básica del sistema de educación universitaria chileno. A día de hoy, se distinguen básicamente tres tipos, universidades miembro del Consejo de Rectores de Chile ( $\mathrm{CRUCH})$, que son aquellas que fueron creadas con anterioridad a la reforma educacional de 1981, y aquellas creadas con posterioridad.

Dentro de las universidades del $\mathrm{CRUCH}$, están las Universidades Públicas, que son propiedad del Estado, y las Universidades Privadas $\mathrm{CRUCH}$, que son universidades confesionales o de grupos de interés regionales. Ambas reciben financiamiento directo del Estado (Aporte Fiscal Directo -AFD-, MECESUP, y otros fondos menor relevancia), aunque las Privadas $\mathrm{CRUCH}$ lo reciben en cantidades y condiciones más ventajosas que muchas instituciones públicas (no están sujetas a la regulación de la Contraloría). Las que no son del $\mathrm{CRUCH}$ se les denomina Universidades Privadas, y no acceden a la financiación directa del Estado, pero sí a las financiación indirecta a través de subvenciones generales para todo el sistema universitario (Aporte Fiscal Indirecto $\mathrm{AFI}^{14}$-, Crédito con Aval del Estado $-\mathrm{CAE}^{15}{ }_{-}$, y fondos concursales para la investigación científica y tecnológica -FONDECYT-).

Esquema 1. Sistema Universitario en Chile por tipo de Universidades.

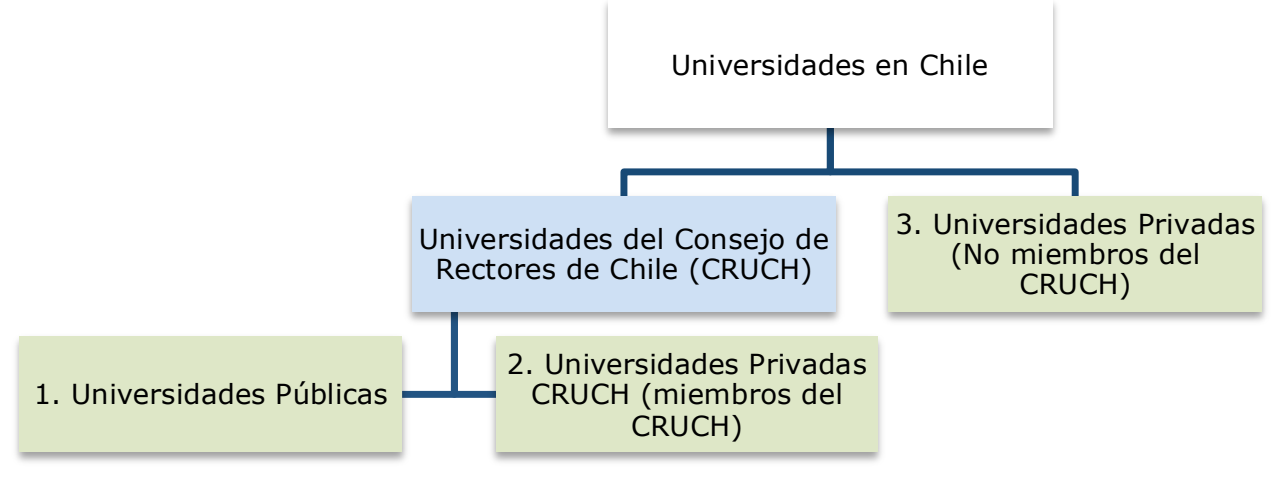

Fuente: Elaboración propia.

\footnotetext{
14 Pago por captura de uno de los estudiantes de los 28.000 mejores puntajes en la Prueba de Selección Universitaria (PSU)

${ }^{15}$ Créditos entregados por la banca privada con el Estado como fiador, que anticipa un porcentaje del préstamo como garantía a los bancos.
} 
Esta arquitectura del sistema ha contribuido a un crecimiento exponencial del número de Universidades Privadas no adscritas al $\mathrm{CRUCH}$, como se observa en el gráfico 1, 35 instituciones Privadas representan el 58,3\% del total de universidades del sistema. El número de Universidades Públicas son 16 y representa el $26,7 \%$ (las universidades de Aysén y O'Higgins no han sido consideradas porque sus alumnos entrarán en 2017), y las Universidades Privadas CRUCH el $15 \%$, con 9 instituciones.

Gráfico 1: Número de universidades por tipo de instituciones en Chile 2015.

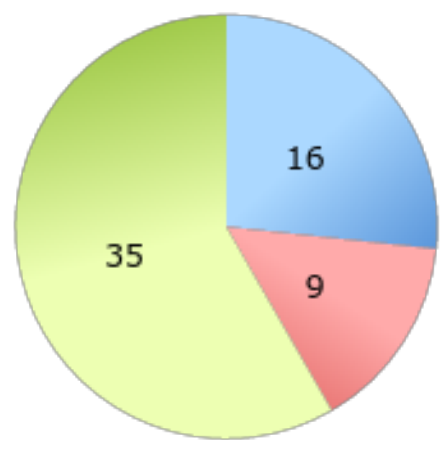

Universidades Públicas

Universidades Privadas CRUCH

Universidades Privadas

Fuente: Elaboración propia con datos oficiales de la Contraloría General de la República de Chile (Contraloría General de la República, 2016: 9).

Este crecimiento exponencial del número de instituciones Privadas, ha tenido su correlato en el número de alumnos que estudian en ellas. De esta forma, el $52,46 \%$ del total de estudiantes matriculados en el sistema universitario chileno estudia en una Universidad Privada, el resto se distribuye en Universidades Públicas $(26,69 \%)$ y Privada CRUCH (20,85\%). También podría leerse como que un $73,3 \%$ del total de alumnos matriculados en las universidades chilenas estudia en una institución Privada.

Gráfico 2: Matrícula de estudiantes según tipo de universidad en Chile 2015 (\%). 


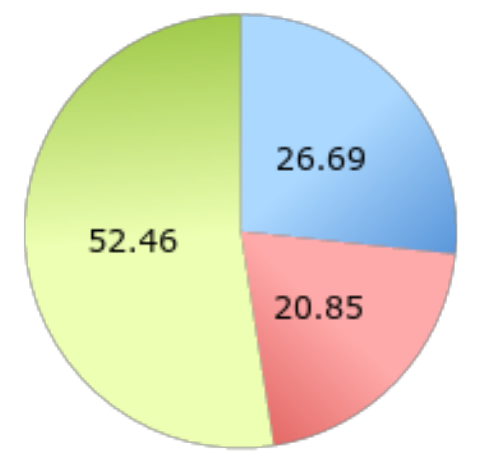

Universidades Públicas

Universidades Privadas CRUCH

$\square$ Universidades Privadas

Fuente: Elaboración propia con datos oficiales de la Contraloría General de la República de Chile (Contraloría General de la República, 2016: 9).

El financiamiento público de la educación superior en Chile es bajo comparado con los países de la OCDE (OCDE, 2015b; OCDE, 2010; Brunner, 2012), ello queda graficado en el informe de la agencia del año 2015, donde Chile aparece con un gasto público en educación superior de un $34,6 \%$, que es la mitad del promedio de los países de la OCDE, con un 69,7\% (OCDE, 2015b: 248). Al no existir una regla uniforme para determinar un financiamiento público "óptimo", y siendo Chile país miembro de la OCDE, por lo menos debería duplicar como porcentaje del PIB su inversión pública en educación superior (OCDE, 2015b).

Tabla 1: Proporciones relativas de gasto público y privado en instituciones educacionales terciarias países seleccionados OCDE 2012 (\%).

\begin{tabular}{|l|c|c|c|c|c|c|c|c|c|c|c|c|c|c|}
\hline & $\begin{array}{c}\text { Co } \\
\text { re } \\
\text { a }\end{array}$ & $\begin{array}{c}\text { Ja } \\
\text { pó } \\
\text { n }\end{array}$ & $\begin{array}{c}\text { Chi } \\
\text { le }\end{array}$ & $\begin{array}{c}\text { EE } \\
\text { U. }\end{array}$ & $\begin{array}{c}\text { R. } \\
\text { Un } \\
\text { ido }\end{array}$ & $\begin{array}{c}\text { Mé } \\
\text { xic } \\
\text { o }\end{array}$ & $\begin{array}{c}\text { Pr } \\
\text { om } \\
\text { edi } \\
\text { o } \\
\text { OC } \\
\text { DE }\end{array}$ & $\begin{array}{c}\text { Es } \\
\text { pa }\end{array}$ & $\begin{array}{c}\text { UE } \\
21\end{array}$ & $\begin{array}{c}\text { Fra } \\
\text { nci } \\
\text { a }\end{array}$ & $\begin{array}{c}\text { Ale } \\
\text { ma } \\
\text { nia }\end{array}$ & $\begin{array}{c}\text { Su } \\
\text { eci } \\
\text { a }\end{array}$ & $\begin{array}{c}\text { No } \\
\text { ru } \\
\text { eg } \\
\text { a }\end{array}$ & $\begin{array}{c}\text { Fin } \\
\text { lan } \\
\text { dia }\end{array}$ \\
\hline $\begin{array}{l}\text { Gasto } \\
\text { Público }\end{array}$ & 29,3 & 34,3 & 34,6 & 37,8 & 56,9 & 69,7 & 69,7 & 73,1 & 78,1 & 79,8 & 85,9 & 89,3 & 96,1 & 96,2 \\
\hline $\begin{array}{l}\text { Gasto } \\
\text { Privado }\end{array}$ & 70,7 & 65,7 & 65,4 & 62,2 & 43,1 & 30,3 & 30,3 & 26,9 & 21,9 & 20,2 & 14,1 & 10,7 & 3,9 & 3,8 \\
\hline
\end{tabular}

Fuente: Elaboración propia con datos OCDE, (OCDE, 2015b).

Como se observa en el gráfico 3, la distribución del financiamiento público, evidencia que no está alineado con la matrícula, que se explica por las restricciones que tienen las Universidades Privadas al financiamiento directo. Así, las 35 Universidades Privadas que concentra el $52,46 \%$ de la matrícula, reciben sólo un $29,1 \%$ de los recursos públicos, que da un promedio por universidad de $0,83 \%$ del presupuesto de educación. Si se hace el mismo ejercicio con las Universidades Públicas y Privadas $\mathrm{CRUCH}$, se observa que las más favorecidas son las 
Privadas $\mathrm{CRUCH}$, pues con su $31,33 \%$ del total de recursos fiscales, las 9 Universidades Privadas $\mathrm{CRUCH}$ reciben en promedio el 3,48\% del presupuesto; a diferencia de las 16 Universidades Públicas, que con su $39,51 \%$ de participación del presupuesto, cada institución recibe en promedio el $2,46 \%$. Esto habla de un sistema descompensado y con claras asimetrías y donde las Universidades Públicas de propiedad estatal no tienen trato especial.

Gráfico 3: Gasto fiscal (34,6\%) por tipo de universidades en Chile 2015 (\%).

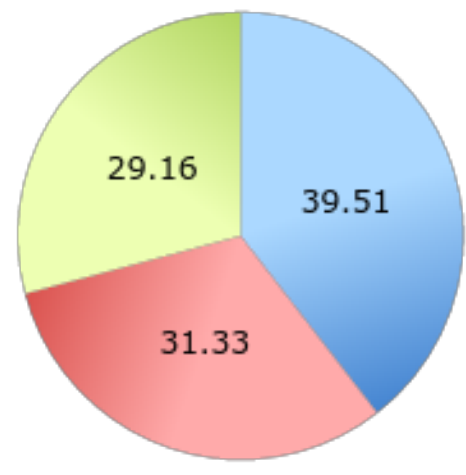

Universidades Públicas

-Universidades Privadas $\mathrm{CRUCH}$

$\square$ Universidades Privadas

Fuente: Elaboración propia con datos oficiales de la Contraloría General de la República de Chile (Contraloría General de la República, 2016: 17).

Esta distribución asimétrica de los recursos se explica por el carácter eminentemente docentes de las Universidades Privadas, que queda demostrado en el gráfico 4 . Su baja participación en los fondos de concurso para investigación científica y tecnológica en Chile (distribuidos por $\mathrm{CONICYT}^{16}$ ), se expresa en un reducido $4,1 \%$ del total de recursos asignados, en contraste con las Universidad Públicas, que concentran un $46,8 \%$, y las Universidades Privadas $\mathrm{CRUCH}$, con un $49,1 \%$.

Gráfico 4: Gasto fiscal en investigación científica y tecnológica por tipo de universidades en Chile 2015 (\%).

${ }^{16}$ Comisión Nacional de Investigación Científica y Tecnológica. 


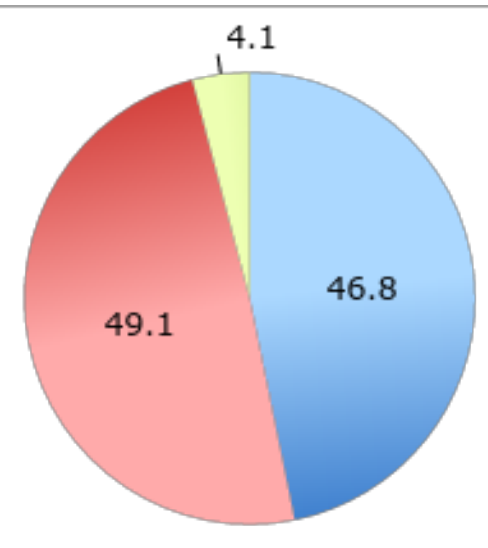

Universidades Públicas

Universidades Privadas CRUCH

Universidades Privadas

Fuente: Elaboración propia con datos oficiales de la Contraloría General de la República de Chile (Contraloría General de la República, 2016: 45).

\section{Descentralización y Universidades regionales.}

Una primera mirada al sistema universitario chileno desde un punto de vista territorial, puede verificar sus asimetrías entre la capital, Santiago, y las regiones. La tabla 2 evidencia la relevancia regional de las Universidades Públicas y Privadas $\mathrm{CRUCH}$, respecto de las Universidades Privadas, pues la mayoría de las primeras dos tiene su Rectorado en regiones. Esta simple constatación permite plantear su mejor posición relativa para comprometerse con estrategias de desarrollo regional, y avanzar a una gestión descentralizada de la capital, lo que permite suponer una más plausible vinculación con sus regiones.

Tabla 2: Ubicación de Rectorado de universidades por tipo de institución 2015.

\begin{tabular}{|l|c|c|c|}
\hline & Públicas & Privadas CRUCH & Privadas \\
\hline Rectoría Santiago & 4 & 1 & 26 \\
\hline Rectoría Regiones & 12 & 8 & 9 \\
\hline
\end{tabular}

Fuente: Elaboración propia.

En sentido contrario, actualmente se constata una tendencia a crear sedes en aquellos lugares con mayor densidad demográfica y actividad económica para capturar mayor matrícula, que se da preferentemente en las Universidades Privadas, pero de las que no han estado exentas las Privadas $\mathrm{CRUCH}$ y, en menor medida, las Públicas. En la práctica, ello ha perjudicado a las regiones más aisladas o pequeñas poblacionalmente, como es el caso de la Región de Aysén. Santiago nuevamente resulta el principal favorecido, concentrando 45 sedes 
extrarregionales, seguido por Bío-Bío (36) y Valparaíso (25) (AUR, 2010). Paradojalmente, ello ha redundado en que algunas Universidades Privadas con Rectorados en regiones, tengan la mayoría de su matrícula en Santiago. Por contraste, las regiones con menos sedes universitarias son Aysén (1), Arica-Parinacota (5), Los Ríos (6), Tarapacá (7) y Atacama (7) (AUR, 2010), que representan regiones extremas y/o con mayor grado de aislamiento.

Si se observa el gráfico 5 , se aprecia que las 4 universidades capitalinas de propiedad Estatal concentran el $39,84 \%$ de la matrícula de las Universidades Públicas ( $10 \%$ promedio por universidad capitalina), repartiéndose el $60,16 \%$ restante de la matrícula entre 12 universidades ( $5 \%$ promedio en universidad regional).

Gráfico 5: Distribución de la matrícula en universidades públicas entre instituciones de Santiago (capitalinas) y Regiones en Chile 2015 (\%).

\section{Santiago (capitalinas) y Regiones en Chile $2015(\%)$.}

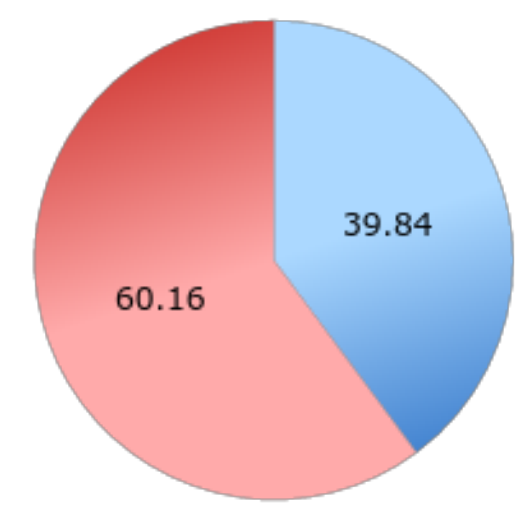

Fuente: Elaboración propia con datos oficiales de la Contraloría General de la República de Chile (Contraloría General de la República, 2016: 84).

Los tres tipos de universidades "compiten" por el Aporte Fiscal Indirecto (AFI), que se basa en la "captura" de los 28.000 mejores puntajes en la Prueba de Selección Universitaria (PSU). De estos recursos, el $77 \%$ fue para las universidades $\mathrm{CRUCH}$ y sólo el $21.5 \%$ para las Universidades Privadas ${ }^{17}$. Un $60 \%$ se quedó en Santiago, repartiéndose el $40 \%$ restante en todas las universidades regionales del país, tanto Públicas, Privadas CRUCH y Privadas (AUR, 2010).

Mirado desde los recursos fiscales orientados a las Universidades Públicas, las diferencias entre Santiago y regiones vuelven a ser

\footnotetext{
${ }^{17}$ El $1.4 \%$ restante fue para los Institutos Profesionales, Fuerzas Armadas y CFT.
} 
significativas. Como se aprecia en el gráfico 6, las Universidades de Santiago, no obstante concentrar el $39,84 \%$ de la matrícula, reciben el $46,85 \%$ del presupuesto fiscal para las Universidades Públicas, que representa un $7 \%$ por sobre su población estudiantil. Las Universidades Públicas regionales reciben un $53,15 \%$ de los recursos. Esto reafirma el carácter centralista en la asignación de recursos y del diseño de los estímulos.

Gráfico 6: Distribución del gasto fiscal en universidades públicas entre instituciones de Santiago (capitalinas) y Regiones en Chile 2015 (\%).

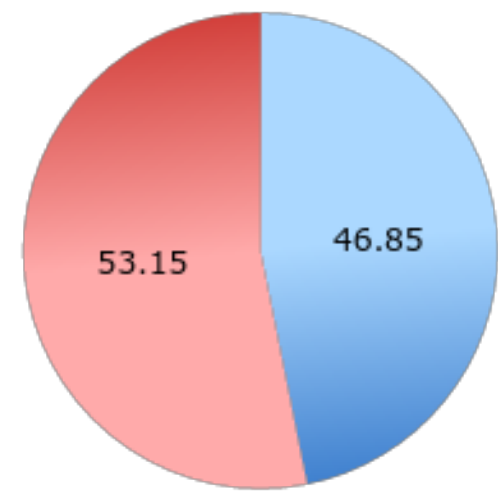

Universidades Públicas Capitalinas Universidades Públicas Regionales

Fuente: Elaboración propia con datos oficiales de la Contraloría General de la República de Chile (Contraloría General de la República, 2016: 84).

Desde una óptica socioeconómica, las universidades del $\mathrm{CRUCH}$ de Santiago (Públicas y Privadas $\mathrm{CRUCH}$ ) presentan una alta concentración de alumnos de nivel socioeconómico medio y en especial alto, con baja presencia de alumnos de niveles socioeconómicos bajos (Valdivieso et al, 2006). En contraste, las universidades regionales del $\mathrm{CRUCH}$ tienen una mayor concentración de alumnos de estratos socioeconómicos bajo y medio bajo (ibídem). Ello permite inferir que los alumnos de mayores recursos de regiones se trasladan a Santiago y los de menores recursos permanecen en sus regiones de origen. Como cabe esperar, por la alta correlación entre altos puntajes de la PSU y los ingresos familiares (Koljatic y Silva, 2010; Beyer, 2009), los puntajes en las pruebas de selección (PSU) de los alumnos de las universidades del $\mathrm{CRUCH}$ en la Región Metropolitana son mayores que los de las universidades regionales, que confirma la selección y captura de los talentos de regiones por parte de la Capital y, por lo tanto, el carácter regresivo del AFI.

Desde el punto de vista de la oferta de programas académicos avanzados (magíster y doctorados), se vuelve a apreciar la asimetría 
entre las regiones y Santiago. Para el año 2008, un 65\% de las maestrías se concentraban en la Región Metropolitana; si se consideran sólo los programas acreditados por el Consejo Nacional de Acreditación (CNA), la región Metropolitana se empina a un 75\% (AUR, 2010). En el caso de los doctorados, el $58 \%$ se concentra en la capital, y si se toma en cuenta sólo los acreditados, Santiago sube a un 67\% (Ibídem).

En el caso de los fondos para el desarrollo de la investigación científica y tecnológica asignada mediante el Concurso Regular de Fondecyt 20032009, las universidades capitalinas capturan el $60 \%$ del total de recursos disponibles (Ibídem). Como plantea la OCDE/BM (2009), se evidencia la falta de políticas públicas de inversión en infraestructura para desarrollar la investigación científica y tecnológica de punta en regiones, en especial, en aquellas áreas donde hay ventajas comparativas.

Por otro lado, se aprecia en el gráfico 7 la desconexión de las Universidades Públicas regionales con su territorio, pues de los recursos que maneja el Gobierno Regional (GORE) para educación, no obstante ser pequeños en el presupuesto global del sistema, la mayor parte se los llevan las Universidades Privadas $\mathrm{CRUCH}$ con un 49,4\%, seguidas por lejos por las Universidades Privadas con un 25,7\%, siendo las que menos reciben recursos del GORE las Universidades Públicas, con un $24,9 \%$.

Gráfico 7: Aportes de Gobiernos Regionales por tipo de universidad en Chile 2015 (\%).

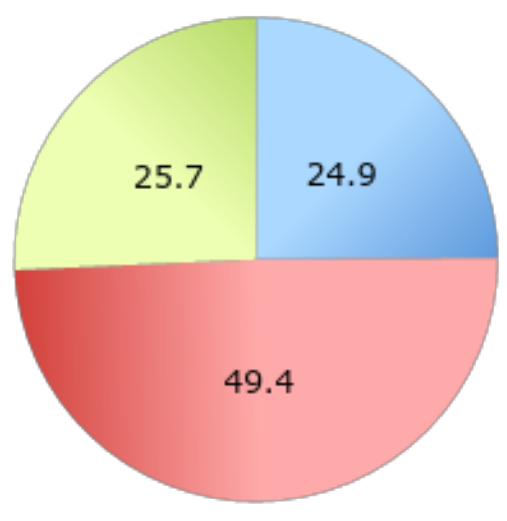

Universidades Públicas Universidades Privadas CRUCH Universidades Privadas

Fuente: Elaboración propia con datos oficiales de la Contraloría General de la República de Chile (Contraloría General de la República, 2016: 61). 
Toda esta evidencia confirma la situación de desventaja en que se encuentran las Universidades Públicas regionales, que permiten afirmar que la estructura actual del sistema universitario chileno y el diseño de los estímulos no estarían favoreciendo la vinculación regional y el impacto de estas instituciones en el desarrollo regional.

\section{Reflexiones finales: Por una nueva vinculación regional universitaria}

Diversas evaluaciones que se han realizado al actual sistema de educación superior chileno, evidencian las dificultades para poder materializar de forma virtuosa esta vinculación entre universidades regionales y los territorios (OCDE/BM, 2009; CAP, 2006; Aequalis, 2012; AUR, 2010; CUECH 2009). Las restricciones del sistema universitario chileno en su estructura de financiamiento, como las dificultades (o falta de incentivos) de las universidades para vincularse a su medio, aparecen como sus principales trabas (OCDE/BM, 2009; AUR, 2010).

Estos son los problemas que debe enfrentar la Universidad de Aysén, pero con una diferencia, que desde su gestación tiene como misión vincularse con su región y comunidad local, y en el logro de aquello será evaluada. Esto podría representar una doble tensión: Por un lado, plantea un requerimiento general al sistema universitario chileno por un financiamiento sensible a consideraciones territoriales que dé cuenta de la diversidad de propósitos y características de las instituciones de regiones. Y por otro lado, la misma génesis reivindicativa regional que le dio vida le exige a esta universidad generar canales permanentes de vinculación con su medio, de forma que pueda dar respuesta y efectos concretos en las necesidades de desarrollo local.

Este doble componente problemático entraña una serie de riesgos. La ausencia de una financiación con criterios territoriales y la continuidad de estímulos indiferentes al emplazamiento de las universidades, puede hacer insostenible financieramente el proyecto de la Universidad de Aysén. Por otro lado, un impacto menor o nulo en el desarrollo regional de la zona, podría hacer perder legitimidad regional al proyecto, y consecuentemente, anular el argumento de que una vinculación con el medio regional sea un objetivo estratégico para potenciar los territorios. Argumento contrario a lo que señalan expertos $u$ organismos internacionales (OCDE, 2009a; Stensaker et al, 2007; Jongbloed et al, 
2008; Santiago et al, 2008; Ferlie et al, 2008; Larsen et al, 2009), que puede verse abonado en la inexistencia de experiencias previas en Chile que avalen los efectos positivos en el desarrollo local de una universidad regional comprometida con su entorno, como señala la literatura.

Diferentes organismos (OCDE, 2009a) y expertos (Stensaker et al, 2007; Jongbloed et al, 2008; Santiago et al, 2008; Ferlie et al, 2008; Larsen et al, 2009) han planteado el impacto positivo que tiene la vinculación de las universidades con su comunidad para el desarrollo regional y local.

Ello permitiría suponer los posibles efectos positivos para el territorio de una universidad de vocación regional en el sistema de educación superior de Chile, como es el caso de la Universidad de Aysén, que debería expresarse en impactos en el desarrollo regional.

Un segundo hecho relevante, deriva de la creciente presión de las universidades regionales para que la asignación de recursos por parte del Estado consideren criterios territoriales (AUR, 2010; Von Baer, 2006 y 2007). Ello permitiría suponer que la creación de la Universidad de Aysén como fruto de una demanda regional, podría tener un impacto en el debate de la reforma a la educación superior para integrar criterios territoriales en la financiación y descentralización universitaria.

\section{Bibliografía}

Aequalis (2012). Propuestas para la Educación Superior: Foro Aequalis y las transformaciones necesarias. Santiago, Chile: Ograma Impresores-Aequalis.

Agrupación de Universidades Regionales (AUR) (2010). Universidades y compromiso regional. Antecedentes, realidades $y$ desafíos. Disponible en línea en fecha de consulta 12/04/2015:

www.auregionales.cl/documentos/documento-finalaur2010.pdf

Atria, F (2014). Derechos sociales y educación: un nuevo paradigma de lo público. Santiago de Chile: Lom Ediciones.

Bachelet, M. (2013). Chile de todos. Programa de Gobierno Michelle Bachelet 2014-2018. Disponible en línea en fecha de consulta 10/02/2016: www.michellebachelet.cl/programa/

Beyer, H. (2009). Igualdad de Oportunidades y Selección a las Universidades, en Puntos de Referencia-Centro de Estudios Públicos 303, 1-10. 
Boisier, S. (2000). Chile: la vocación regionalista del gobierno militar. Revista Eure 77(26), 81-107.

Brunner, J. J. (2012). Educación Superior en Iberoamérica: Informe 2011. Santiago, Chile: CINDA-Universia.

Brunner, J. J. (1986). Informe sobre la educación superior en Chile. Santiago, Chile: Flacso.

Clark, B. (1998). Creating Entrepreneurial Universities. Organizational Pathways of Transformation. Oxford, Inglaterra: Pergamon Press.

Comisión Asesora Presidencial en Descentralización y Desarrollo Regional (CAPDDR) (2014). Informe Comisión Asesora Presidencial en Descentralización y Desarrollo Regional. Propuesta de política de Estado y agenda para la descentralización y el desarrollo territorial de Chile. Santiago, Chile: Presidencia de la República de Chile. Disponible en línea en fecha de consulta 22/03/2016:

https://prensa.presidencia.cl/lfi-content/otras/informescomisiones/InformeDescentralizacion.pdf

Consejo Asesor Presidencial para la Educación Superior (CAP) (2008). Los desafíos de la educación superior chilena. Informe del Consejo Asesor Presidencial para la Educación Superior. Disponible en línea en fecha de consulta 08/05/2015: www.ubiobio.cl/web/descargas/InformeCAPfinal.pdf

Consorcio de Universidades del Estado de Chile (CUECH) (2009). Nuevo Trato con el Estado: Fortaleciendo la educación superior estatal. Disponible en línea en fecha de consulta 22/05/2015:

http://www.uchile.cl/uchile/download.jsp?document=51009\&proper ty $=$ attachment\&index $=0$ \&content $=$

Contraloría General de la República de Chile (2016). Financiamiento fiscal a la educación superior 2015. Santiago, Chile: Contraloría General de la República de Chile.

Echeverría, R. R. (1993). El búho de Minerva. Introducción a la filosofía moderna. Santiago, Chile: Dolmen.

Erickson, F. y Mohatt, G. (1982). Cultural organization of participation structures in two classrooms of Indian students. En G. Spindler (Ed.), Doing the ethnography of schooling: Educational anthropology in action (pp. 132-174). New York: Holt, Rinehart, and Winston.

Ferlie, E.; Musselin C. y Andresani G. (2008). The Steering of Higher Education Systems: A Public Management Perspective. Higher Education 56(3), 325-348.

Fontana, A. y Frey, J. (2005). The Interview, from neutral stance to political involvement. En N. Denzin y S. Lincoln (Comp). The Sage Handbook of Qualitative Research (695-727). London: Sage. 
Gornitzka, A.; Kyvik S. y Larsen I. M. (1998). The Bureaucratisation of Universities. Minerva 36(1), 21-47.

Instituto Nacional de Estadísticas (INE) (2012). Compendio_estadístico 2012. Santiago, Chile: Instituto Nacional de Estadísticas.

Jongbloed, B.; Enders J. y Salerno C. (2008). Higher Education and its Communities: Interconnections, Interdependencies and a Research Agenda. Higher Education 56, 303-324.

Kvale, S. (1996). Interviews: An introduction to qualitative research interviewing. Thousand Oaks, California: Sage.

Kim, L. (2001). Conflicts Within Swedish Higher Education Policy. International Higher Education 23. Disponible en línea en fecha de consulta 15/04/2015:

www.bc.edu/bc org/avp/soe/cihe/newsletter/News23/text013.htm

Koljatic, M. y Silva M. (2010). Algunas reflexiones a siete años de la implementación de la PSU. Revista Estudios Públicos 124, 125-146.

Larsen, I. M.; Maassen P. y Stensaker B. (2009). Four Basic Dilemmas in University Governance Reform. Higher Education Management and Policy 21(3), 41-58.

Maassen, P. (2008). The Modernisation of European Higher Education. National Policy Dynamics. En A. Amaral, I. Bleiklie y C. Musselin (eds.). From Governance to Identity, Festschrift for Mary Henkel. Dordrecht, Holland: Springer Science (pp. 95-113).

Martinic, M. (1973). Panorama de la colonización en Tierra del Fuego entre 1881 y 1900 . Anales del Instituto de la Patagonia, 4(1-3), 569.

Ministerio de Educación (MINEDUC) (2015a). Una comunidad que sueña la Universidad Estatal de Aysén. Propuestas ciudadanas de una region que dialoga. Coyhaique, Región de Aysén: MINEDUC.

Ministerio de Educación (MINEDUC) (2015b). Sentando las bases para habitar de otro modo el planeta. Propuesta de la Comisión Ejecutiva para crear la Universidad Estatal de Aysén. Coyhaique, Región de Aysén: MINEDUC.

Montecinos, E. (2011). Democracia participativa y presupuesto participativo en Chile: ¿Complemento subordinación a las instituciones representativas locales?. Revista de ciencia política (Santiago), 31(1), 63-89. Disponible en línea en fecha de consulta 01/03/2015:

http://www.scielo.cl/scielo.php?script=sci_arttext\&pid=S0718-

090X2011000100004\&lng=es\&tlng=es.\%2010.4067/S0718090X2011000100004 
Organización para la Cooperación y el Desarrollo Económico (OCDE) (2015a). In it together. Why less inequality benefits all. Paris, France: OECD Publishing.

OCDE (2015b). Education at a glance 2015. OECD indicators. Paris, France: OECD Publishing.

OCDE (2010). Panorama de la educación 2010 Indicadores de la OCDE. Madrid, España: OCDE- Santillana Educación S.L.

OCDE (2009a). Educación superior en el desarrollo regional y de ciudades. Paris, Francia: OCDE.

OCDE (2009b). Estudios Territoriales de la OCDE: Chile 2009. Santiago, Chile: Ministerio del Interior.

OCDE y Banco Mundial (BM) (2009). La educación superior en Chile. Santiago, Chile: OCDE/BM/Ministerio de Educación de Chile.

O'Donnell, Guillermo (1996). El estado burocrático-autoritario. Triunfos, derrotas y crisis. Buenos Aires, Argentina: Editorial de Belgrano.

Programa de las Naciones Unidas para el Desarrollo (PNUD) (2015). Panorama general. Informe sobre Desarrollo Humano 2015. Trabajo al servicio del desarrollo humano. Nueva York, Estados Unidos: PNUD.

PNUD (2013). La desigualdad incita la protesta social en América Latina, señala un nuevo informe del PNUD. Disponible en línea en fecha de consulta 20/03/2016:

http://www.undp.org/content/undp/es/home/presscenter/pressrele ases/2013/04/16/la-desigualdad-incita-la-protesta-social-enamerica-latina-se-ala-un-nuevo-informe-del-pnud.html

Rodríguez, J. y Busso G. (2009). Migración interna y desarrollo en América Latina entre 1980 y 2005 . Un estudio comparativo con perspectiva regional basado en siete países. Santiago, Chile: Publicación de las Naciones Unidas.

Rosso, P. P. y Reyes C. (15/01/2012). El nuevo ranking que divide a las universidades en cuatro categorías. Diario El Mercurio, (D8-D9).

Santiago, P.; Tremblay, K.; Basri, E. y Arnal E. (2008). Tertiary Education for the Knowledge Society Volume 1. Paris, Francia: OECD.

Schwartz, H y Hacobs, J (1984). Sociología cualitativa. Método para la reconstrucción de la realidad. México Df, México: Editorial Trillas.

Stensaker, B.; Enders J. y de Boer H. (2007). Comparative Analysis, Final report of the project: The Extent and Impact of Governance Reform across Europe. Brussels, Belgium: European Commission.

Subsecretaría de Desarrollo Regional (SUBDERE) (2000). Diagnóstico sobre el Proceso de Descentralización en Chile. Santiago, Chile: 
SUBDERE/División de Evaluación y Cuentas Públicas. Disponible en línea en fecha de consulta 20/02/2016:

http://www.subdere.gov.cl/sites/default/files/documentos/articles65837 recurso $1 . p d f$

Toulmin, S. (2001). Cosmópolis: el trasfondo de la modernidad, Barcelona: Península.

Universidad de Aysen (2016). Proyecto de estatutos de la Universidad de Aysen. Coyhaique, Región de Aysén: Universidad de Aysén. Disponible en línea en fecha de consulta 20/02/2016: http://www.uaysen.cl/estatutos/

Valenzuela, E. (2015). Territorios rebeldes por la autonomía en el ciclo de protestas y transformaciones políticas en Chile. En Valenzuela $\mathrm{E}$. (editor), Territorios rebeldes. Autonomías versus presicracia centralista. Santiago, Chile: Ediciones Universidad Alberto Hurtado (pp. 13-20).

Von Baer, H. (2006). Descentralización y desarrollo local/regional. La tarea pendiente del modelo "chilensis" de desarrollo. Agenda Pública 5(10), 2-16.

Von Baer, H. (2007). Descentralización: imperativo para el desarrollo humano y territorial equilibrado con gobernabilidad, en un contexto de globalización. Una mirada desde Chile. Cuadernos de Administración 36-37, 12-40. 